\title{
Esophageal Kaposi Sarcoma
}

National Cancer Institute

\section{Source}

National Cancer Institute. Esophageal Kaposi Sarcoma. NCI Thesaurus. Code C5706.

A Kaposi sarcoma arising from the esophagus. 